\title{
Diagnostic value of urinary-to-serum human epididymis protein 4 ratio in ovarian cancer
}

\author{
QUMING FAN $^{1}$, GUANGCHENG LUO $^{1 *}$, TINGTING YI ${ }^{1}$, QIANG WANG $^{1}$, DONGSHENG WANG $^{1}$, \\ GUOYUAN ZHANG ${ }^{1}$, XINGLIANG JIANG ${ }^{1}$ and XIAOLAN GUO ${ }^{1,2}$ \\ ${ }^{1}$ Department of Clinical Laboratory, Affiliated Hospital of North Sichuan Medical College; \\ ${ }^{2}$ Translational Medicine Research Center, North Sichuan Medical College, Nanchong, Sichuan 637000, P.R. China
}

Received February 14, 2017; Accepted April 27, 2017

DOI: $10.3892 /$ br.2017.913

\begin{abstract}
Human epididymis protein 4 (HE4) is one of the best-known tumor markers for ovarian cancer (OC). Emerging evidence indicates that the evaluation of serum HE4 (S-HE4) levels may be problematic when patients have chronic kidney disease (CKD). Assaying urine for HE4 levels is non-invasive alternative for the diagnosis of OC. However, whether the combined detection of S- and urinary HE4 (U-HE4) levels distinguishes $\mathrm{OC}$ from $\mathrm{CKD}$ remains unknown. To investigate this issue, the present study recruited 31 female patients with OC, 38 female patients with CKD, and 36 healthy control (HC) females. Serum and urine samples were preoperatively collected for HE4 level detection. Receiver operating characteristic (ROC) curves were constructed to assess the diagnostic performance of S-HE4 level, U-HE4 level and the ratio of urinary-to-serum HE4 level (R-HE4). Data from the current study indicated that serum HE4 levels in the OC and CKD groups were significantly higher than that in the $\mathrm{HC}$ group. The U-HE4 level in the OC group was significantly higher than that in the CKD and $\mathrm{HC}$ groups. The highest R-HE4 was observed in the HC group, followed by the OC group, and the lowest R-HE4 was observed in the CKD group. ROC analysis demonstrated that the R-HE4 was useful in differentiating OC from CKD and HC. Based on the diagnostic interval of optimal cut-off values from 36.85 to 96.15 , the sensitivity and specificity of R-HE4 in differentiating OC patients from non-OC patients were 82.6 and $85.4 \%$, respectively. Thus, the combined detection of S- and U-HE4 levels facilitates the diagnosis of OC, and R-HE4 is an effective marker for differentiating OC from CKD.
\end{abstract}

Correspondence to: Dr Quming Fan, Department of Clinical Laboratory, Affiliated Hospital of North Sichuan Medical College, 63 Wenhua Road, Nanchong, Sichuan 637000, P.R. China E-mail: 421287434@qq.com

Key words: human epididymis protein 4, ovarian cancer, chronic kidney disease, urine

\section{Introduction}

Ovarian cancer (OC) is the most common cause of mortality among women with gynecologic cancer worldwide (1). Although this tumor type may develop at young ages, the majority of the cases occur in postmenopausal women (2). Since the disease presents with non-specific symptoms, approximately $70 \%$ of patients with OC are not diagnosed until the disease has reached an advanced stage (3). Patients with high-grade OC often have poor prognosis and a high mortality rate (4). Therefore, early diagnosis of OC is a key factor in improving patient survival. Currently, tumor markers, such as the human epididymis protein 4 (HE4) (5) and carbohydrate antigen-125 (CA-125) (6), and the risk of ovarian malignancy algorithm (ROMA) and risk malignancy index (RMI) (7-9) are important tools for the differential diagnosis of patients with abdominopelvic masses.

HE4 is a member of the four-disulfide core family that comprises a heterogeneous group of small acid- and heat-stable proteins of divergent function (5). Over the past decade, HE4 has gained widespread use as an effective tumor marker in the diagnosis of OC. Numerous clinical studies have demonstrated significant elevations of serum HE4 levels in patients with gynecological cancer and have confirmed that HE4 levels may be used as a biomarker for OC with higher specificity than the widely used CA-125 (4,7,8,10-12). A previous study observed that HE4 had sensitivity of $72.9 \%$ and specificity of $95 \%$ in the differential diagnosis of OC and benign ovarian masses (13).

Although HE4 is a valuable marker in OC diagnosis, under certain circumstances, the evaluation of serum HE4 levels may be problematic when patients suffer from additional conditions (4). Abnormal HE4 concentrations are detected in certain nonmalignant diseases, causing difficulties in the differential diagnosis of OC. Furthermore, age, menopause status, and smoking habits directly affect serum HE4 levels; therefore, these conditions should be considered in patients who present with abnormal HE4 levels. Recent studies reported that serum HE4 concentrations significantly increase in patients with chronic kidney disease (CKD), renal failure and heart failure (14-16). In the study by Nagy et al (14), increased HE4 levels were measured in patients with early stage CKD, indicating that the serum HE4 level is significantly affected by the estimated glomerular 
filtration rate (eGFR). Lv et al (17) demonstrated that patients with chronic renal deficiency exhibited elevated serum HE4 levels that were significantly higher than those of patients with benign gynecological diseases. These data indicate that serum HE4 concentrations may be affected by variable demographical factors or by non-malignant diseases. Thus, serum HE4 levels show a high false-positive rate in the differential diagnosis of $\mathrm{OC}$, with the main factor being the presence of CKD (4).

Recent studies have suggested that urine assays are a non-invasive alternative for the evaluation of HE4 levels. Urinary HE4 levels in patients with OC are significantly higher than those in healthy women or patients with benign diseases (10). The ratio between urinary HE4 and urinary creatinine facilitates the differential diagnosis of benign and malignant ovarian tumors (18). Furthermore, the combination of HE4 with CA-125 or eGFR is helpful for discriminating healthy controls from patients with OC (19).

The question of whether the combined detection of serum and urinary HE4 levels distinguishes OC from CKD remains unknown. Therefore, the aim of the present study was to evaluate the diagnostic efficacy of the combined detection of serum and urinary HE4 levels in differentiating OC from CKD.

\section{Materials and methods}

Ethical approval. The present study was approved by the Medical Ethics Committee of the Affiliated Hospital of North Sichuan Medical College (Nanchong, China) and each participant provided written informed consent.

Recruitment of patients. In the present study, 31 patients with OC (whose diagnosis was confirmed by postoperative pathological findings), 38 female patients with CKD and 36 healthy control (HC) females were consecutively recruited from the Affiliated Hospital of North Sichuan Medical College between August 2014 and July 2016. The mean age of patients in the OC, CKD, and HC groups was $53 \pm 12$ years (range, 16-74 years), 55 \pm 13 years (range, 27-76 years), and $52 \pm 17$ years (range, 24-83 years), respectively. All participants within each group were age-matched. In the OC group, there were 11 cases of serous papillary carcinoma, 9 cases of low-grade serous carcinoma, 6 cases of endometrioid carcinoma, 4 cases of high-grade serous carcinoma, and 1 case of malignant germ cell tumor. According to the OC staging guidelines of the International Federation of Gynecologists and Obstetricians (20), there were 3 cases of stage I, 8 cases of stage II, 17 cases of stage III, and 3 cases of stage IV. Eleven patients were premenopausal, while 20 patients were postmenopausal. Diagnoses of CKD were reconfirmed by nephrologists according to Kidney Disease Improving Global Outcomes guidelines (21). The participants in the CKD group exhibited various types of chronic disease, such as hypertension, cardiovascular disease, type 2 diabetes mellitus, hyperlipidemia, autoimmune disease, peripheral artery disease and renal dysfunction (eGFR $<90 \mathrm{ml} / \mathrm{min} / 1.73 \mathrm{~m}^{2}$ ). Participants in the control group had normal eGFR values (eGFR>90 ml/min/1.73 $\mathrm{m}^{2}$ ) and were free of benign and malignant gynecological diseases.
Sample collection. Serum samples $(3 \mathrm{ml})$ were obtained by venipuncture and collected into vacuum tubes to clot. Samples were then centrifuged at $500 \mathrm{x}$ g for $5 \mathrm{~min}$ at room temperature. Urine samples were simultaneously collected for HE4 level detection. All samples were obtained preoperatively at primary diagnosis. The serum and urine samples were cryopreserved $\left(-80^{\circ} \mathrm{C}\right)$ until HE4 level analysis.

Measurement of HE4 level. Serum HE4 (S-HE4) and urinary HE4 (U-HE4) concentrations were measured by electrochemiluminescent immunoassay on a Cobas 800 e602 (Roche Diagnostics, Shanghai, China). The HE4 cut-off value was $140 \mathrm{pmol} / \mathrm{l}$ and the HE4 measurement range was 15-1,500 pmol/1. Samples with HE4 concentrations greater than the measurement range $(1,500 \mathrm{pmol} / \mathrm{l})$ were re-measured following dilution according to the manufacturer's instructions.

Other variables. The serum CA-125 concentration was measured by electrochemiluminescent immunoassay on a Cobas 800 e602. The serum creatinine concentration was measured by enzymatic assay on a AU5800 AU chemistry autoanalyzer (Beckman Coulter, Inc., Shanghai, China) and the serum cystatin concentration was measured using a particle-enhanced turbidimetric immunoassay on the AU5800 AU chemistry autoanalyzer. The value of eGFR was calculated according to the CKD epidemiology collaboration equation (22). Additional clinical and demographic characteristics, and patient laboratory data were obtained by review of medical records.

Statistical analysis. As all of the continuous variables had skewed distributions, the median and range were used to describe these variables. Differences between groups were evaluated using the Kruskal-Wallis test and Mann-Whitney $\mathrm{U}$ test. A receiver operating characteristic (ROC) curve was constructed to assess specificity, sensitivity, and the area under the curve (AUC) with a 95\% confidence interval (CI). The optimal cut-off value for diagnosis was selected by maximizing Youden's index (the sum of sensitivity and specificity) and minimizing the overall error [square root of the sum $\left.(1 \text {-sensitivity })^{2}+(1 \text {-specificity })^{2}\right] . \mathrm{P}<0.05$ was considered to indicate a statistically significant difference and data analyses were performed using SPSS 17.0 statistical software (SPSS, Inc., Chicago, IL, USA).

\section{Results}

Comparisons of variables among the $O C, C K D$ and $H C$ groups. S-and U-HE4 levels and ratios of urinary-to-serum HE4 (R-HE4) levels were analyzed in the OC, CKD and HC groups. As presented in Table I and Fig. 1, S-HE4 levels in the $\mathrm{OC}$ and CKD groups were significantly higher than those in the $\mathrm{HC}$ group $(\mathrm{P}<0.001)$. No significant difference in $\mathrm{S}-\mathrm{HE} 4$ levels was identified between the OC and CKD groups. U-HE4 levels in the OC group were significantly higher than those in the CKD and $\mathrm{HC}$ groups $(\mathrm{P}<0.001)$. No significant difference in U-HE4 levels was identified between the CKD and HC groups. The R-HE4 was observed to be significantly different between the OC, CKD and HC groups $(\mathrm{P}=0.010)$. The R-HE4 in the OC group was significantly higher than that in the CKD 
Table I. Comparison of variables among the OC, CKD and $\mathrm{HC}$ groups.

\begin{tabular}{lccc}
\hline Variable & OC $(\mathrm{n}=31)$ & CKD $(\mathrm{n}=38)$ & HC $(\mathrm{n}=36)$ \\
\hline S-HE4 $(\mathrm{pmol} / \mathrm{l})$ & $439(43-4927)^{\mathrm{a}}$ & $670.1(41-3212)^{\mathrm{a}}$ & $45.65(28-76)$ \\
U-HE4 $(\mathrm{pmol} / \mathrm{l})$ & $28,560(6752-98740)^{\mathrm{a}, \mathrm{b}}$ & $5790(1,621-37,024)$ & $6,573(3,220-12,542)$ \\
R-HE4 & $71.091(14.7-309.7)^{\mathrm{a}, \mathrm{b}}$ & $8.7(1.7-149.5)^{\mathrm{a}}$ & $134.4(71.9-281.3)$ \\
Creatinine $(\mu \mathrm{mol} / \mathrm{l})$ & $56.7(37.9-95.3)^{\mathrm{b}}$ & $279.9(69.8-980.2)^{\mathrm{a}}$ & $48.2(34.2-76.3)$ \\
eGFR $\left(\mathrm{ml} / \mathrm{min} / 1.73 \mathrm{~m}^{2}\right)$ & $102.9(52.2-127.3)^{\mathrm{b}}$ & $18.2(3.0-78.3)^{\mathrm{a}}$ & $116.8(70.1-138.3)$ \\
Serum cystatin C $(\mathrm{mg} / \mathrm{l})$ & $0.71(0.5-1.31)^{\mathrm{b}}$ & $2.91(1.15-7.66)^{\mathrm{a}}$ & $0.66(0.38-0.94)$
\end{tabular}

${ }^{\mathrm{a}} \mathrm{P}<0.05$ vs. HC; ${ }^{\mathrm{b}} \mathrm{P}<0.05$ vs. CKD. OC, ovarian cancer; CKD, chronic kidney disease; HC, healthy control; HE4, human epididymis protein 4 ; $\mathrm{S}$-, serum; U-, urine; R-HE4, ratio of urinary-to-serum HE4 level; eGFR, estimated glomerular filtration rate.

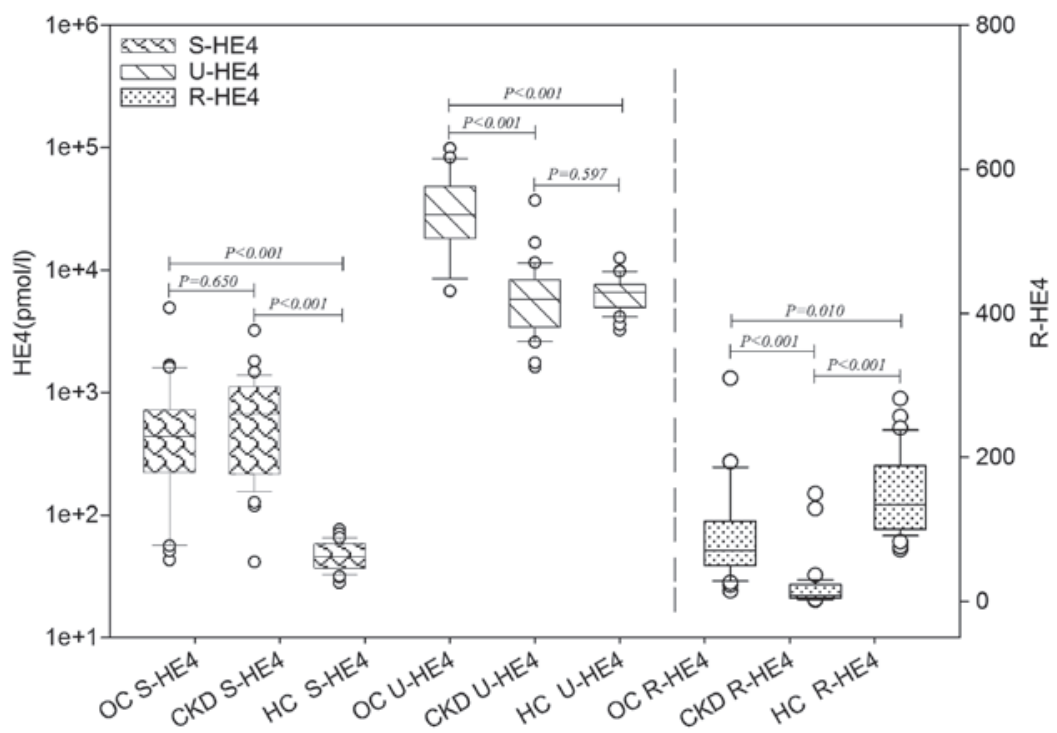

Figure 1. Levels of HE4 in the different patient categories. The S-HE4 levels in the OC and CKD groups are significantly higher than those in the HC group $(\mathrm{P}<0.001)$. The U-HE4 level in the OC group is significantly higher than that in the CKD and HC groups $(\mathrm{P}<0.001)$. Significant differences were observed in R-HE4 among the $\mathrm{OC}$ and $\mathrm{CKD}$ patients, and $\mathrm{HC}$ subjects (HC>OC>CKD; $\mathrm{P}<0.01$ ). HE4, human epididymis protein 4; S-, serum; OC, ovarian cancer; $\mathrm{CKD}$, chronic kidney disease; HC, healthy control; U-, urine; R-HE4, ratio of urinary-to-serum HE4 level.

group $(\mathrm{P}<0.001)$ and significantly lower than that in the $\mathrm{HC}$ group $(\mathrm{P}<0.001)$. Furthermore, the serum levels of creatinine and cystatin $\mathrm{C}$ in the $\mathrm{CKD}$ group were significantly higher than those in the OC and HC groups $(\mathrm{P}<0.001)$. The eGFR value in the CKD group was significantly lower than that in the $\mathrm{OC}$ and $\mathrm{HC}$ groups $(\mathrm{P}<0.001)$.

Diagnostic performance of HE4 in differentiating OC from $H C$. ROC curves were constructed to investigate the diagnostic performance of S- and U-HE4 levels and R-HE4 for distinguishing OC from HC. As shown in Fig. 2 and Table II, the AUC of S-HE4 was 0.955 (95\% CI, 0.907-1.004), which provided an optimal cut-off value of $88.65 \mathrm{pmol} / 1$, sensitivity of $87.1 \%$ and specificity of $100.0 \%$ in distinguishing OC from HC. The AUC value of U-HE4 was 0.959 (95\% CI, 0.915-1.003), which provided an optimal cut-off value of $14,116 \mathrm{pmol} / \mathrm{l}$, sensitivity of $83.9 \%$, and specificity of $100.0 \%$ in distinguishing OC from HC. Consistent with the results of S- and U-HE4 levels, the R-HE4 was useful for differentiating $\mathrm{OC}$ from HC. The AUC of R-HE4 was 0.815 (95\% CI, 0.705-0.926), which provided an optimal cut-off value of 96.15 , sensitivity of $71.0 \%$ and specificity of $88.9 \%$ in separating OC from HC.

Diagnostic performance of HE4 in differentiating OC from $C K D$. As previously stated, no significant difference in S-HE4 levels were observed between OC and CKD. Consequently, S-HE4 levels did not assist with differentiating OC from CKD (AUC $=0.416 ; 95 \%$ CI, 0.277-0.555, $12.9 \%$ sensitivity and $94.7 \%$ specificity). Conversely, U- and R-HE4 levels were useful in differentiating OC from CKD. As presented in Fig. 3 and Table III, the AUC of U-HE4 levels was 0.948 (95\% CI, 0.900-0.996), which provided an optimal cut-off value of $13,586 \mathrm{pmol} / \mathrm{l}$, sensitivity of $83.9 \%$, and specificity of $94.7 \%$ in distinguishing OC from CKD. Similarly, the AUC of R-HE4 was 0.935 (95\% CI, 0.869-1.001), which provided an optimal cut-off value of 36.85 , sensitivity of $90.3 \%$, and specificity of $94.7 \%$ in distinguishing OC from CKD.

Diagnostic performance of $\mathrm{HE} 4$ in differentiating $C K D$ from $H C$. ROC curves were used to investigate the diagnostic performance of S- and U-HE4 levels and R-HE4 
Table II. Diagnostic performance of HE4 in differentiating patients with ovarian cancer from healthy control subjects.

\begin{tabular}{lccc}
\hline Variable & Serum HE4 & Urine HE4 & R-HE4 \\
\hline Area under the curve & 0.955 & 0.959 & 0.815 \\
P-value & $<0.001$ & $<0.001$ & $<0.001$ \\
95\% CI & $0.907-1.004$ & $0.915-1.003$ & $0.705-0.926$ \\
Cut-off value & 88.65 & 14116 & 96.15 \\
Sensitivity (\%) & 87.1 & 83.9 & 71.0 \\
Specificity (\%) & 100.0 & 100.0 & 88.9 \\
Youden's index (\%) & 87.1 & 83.9 & 59.9
\end{tabular}

HE4, human epididymis protein 4; R-HE4, ratio of urinary-to-serum HE4 level; CI, confidence interval.

Table IV. Diagnostic performance of HE4 in differentiating chronic kidney disease patients from healthy control subjects.

\begin{tabular}{lccc}
\hline Variable & Serum HE4 & Urine HE4 & R-HE4 \\
\hline Area under the curve & 0.984 & 0.399 & 0.972 \\
P-value & $<0.001$ & 0.133 & $<0.001$ \\
95\% CI & $0.952-1.016$ & $0.265-0.532$ & $0.933-1.011$ \\
Cutoff value & 97.15 & 10062 & 48.54 \\
Sensitivity (\%) & 97.4 & 15.8 & 94.7 \\
Specificity (\%) & 100.0 & 97.2 & 100.0 \\
Youden's index (\%) & 97.4 & 13.0 & 94.7 \\
\hline
\end{tabular}

HE4, human epididymis protein 4; R-HE4, ratio of urinary-to-serum HE4 level; CI, confidence interval.

in distinguishing CKD from HC. As shown in Fig. 4 and Table IV, the AUC of S-HE4 levels was 0.984 (95\% CI, $0.952-1.016)$, which provided an optimal cut-off value of $94.15 \mathrm{pmol} / \mathrm{l}$, sensitivity of $97.4 \%$, and specificity of $100.0 \%$ in distinguishing CKD from HC. Conversely, U-HE4 levels were of little diagnostic value for differential diagnosis between CKD and HC (AUC $=0.399,95 \%$ CI, 0.265-0.532, $15.8 \%$ sensitivity and $97.2 \%$ specificity). Similar to the results for S-HE4, the AUC of R-HE4 was 0.972 (95\% CI, 0.933-1.011), which provided an optimal cut-off value of 48.54, sensitivity of $94.7 \%$, and specificity of $100.0 \%$ in distinguishing CKD from $\mathrm{HC}$.

Diagnostic performance of $\mathrm{R}-\mathrm{HE} 4$ in differentiating $O C$ patients from non-ovarian cancer objects. As previously described, the R-HE4 was useful for differentiating OC from CKD and $\mathrm{HC}$ with the optimal cut-off values of 36.85 and 96.15 , respectively. The diagnostic performance of R-HE4 was further analyzed based on the diagnosis interval of 36.85 to 96.15 . As shown in Fig. 5, the R-HE4 provided a sensitivity value of $82.6 \%$ and a specificity value of $85.4 \%$ in differentiating $\mathrm{OC}$ patients from non-ovarian cancer objects (including CKD and $\mathrm{HC}$ ).
Table III. Diagnostic performance of HE4 in differentiating patients with ovarian cancer from patients with chronic kidney disease.

\begin{tabular}{lccc}
\hline Variable & Serum HE4 & Urine HE4 & R-HE4 \\
\hline Area under the curve & 0.416 & 0.948 & 0.935 \\
P-value & 0.232 & $<0.001$ & $<0.001$ \\
95\% CI & $0.277-0.555$ & $0.900-0.996$ & $0.869-1.001$ \\
Cut-off value & 1530 & 13586 & 36.85 \\
Sensitivity (\%) & 12.9 & 83.9 & 90.3 \\
Specificity (\%) & 94.7 & 94.7 & 94.7 \\
Youden's index (\%) & 7.6 & 78.6 & 85.1 \\
\hline
\end{tabular}

HE4, human epididymis protein 4; R-HE4, ratio of urinary-to-serum HE4 level; CI, confidence interval.

\section{Discussion}

To the best of the authors' knowledge, this is the first study to investigate the diagnostic performance of R-HE4 in the diagnosis of OC. In the present study, R-HE4 demonstrated significant differences between the $\mathrm{OC}$ and $\mathrm{CKD}$ patients, and healthy controls $(\mathrm{HC}>\mathrm{OC}>\mathrm{CKD} ; \mathrm{P}<0.01)$. In addition, $\mathrm{ROC}$ analysis indicated that R-HE4 was useful for differentiating OC from CKD and HC, with the optimal cut-off values of 36.85 and 96.15 , respectively, with sensitivity of $82.6 \%$ and specificity of $85.4 \%$ in differentiating OC patients from non-cancer objects (including CKD and HC group).

$\mathrm{OC}$ is a heterogeneous group of diseases that exhibits various pathological characteristics and clinical manifestations (1). Early diagnosis is critical for the management and prognosis of OC. An increasing number of studies have demonstrated the important role of HE4 as a tumor marker in the diagnosis of OC (5). For example, a large study demonstrated that S-HE4 has a higher sensitivity and specificity in the diagnosis of OC when compared with serum CA-125 (23). However, the evaluation of S-HE4 levels may be problematic when patients suffer from additional conditions, such as CKD (17), heart failure (4), and breast (24) and lung (25) cancer. Thus, the accuracy of S-HE4 in OC diagnosis remains a challenge. Research has shown that S-HE4 levels demonstrate a high false-positive rate in the differential diagnosis of $\mathrm{OC}$, with the main factor being the presence of CKD (4). Therefore, the differential diagnoses of CKD should be considered for patients with elevated S-HE4 levels.

HE4, like various other tumor biomarkers, is detected in the urine and used as a potentially non-invasive diagnostic tool for OC diagnosis $(10,18)$. However, previous studies have not investigated the combination detection efficacy of $\mathrm{S}$-and U-HE4 levels. In the present study, S- and U-HE4 levels and R-HE4 were analyzed in OC and CKD patients, and HCs. The results indicated that the S-HE4 level in the OC and CKD groups was significantly higher than that in the $\mathrm{HC}$ group $(\mathrm{P}<0.001)$, and that no significant difference regarding $\mathrm{S}-\mathrm{HE} 4$ levels was identified between the OC and CKD groups. These observations were consistent with those of the study by Lv et al (17), which demonstrated that S-HE4 levels in OC and CKD patients significantly increased in comparison to the 


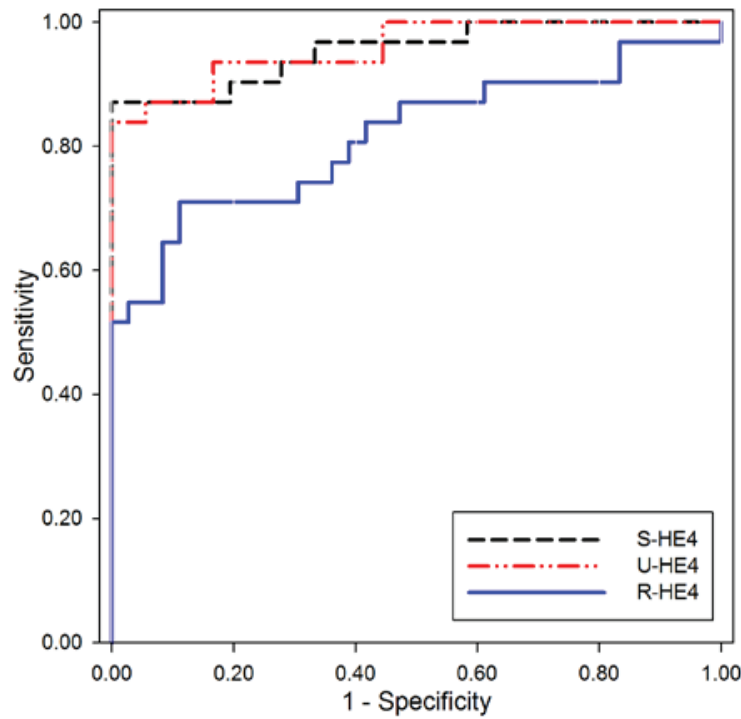

Figure 2. Receiver operating characteristic curves of HE4 for differentiating patients with ovarian cancer from healthy control subjects. The area under the curve of S-HE4, U-HE4, and ratio of urinary-to-serum HE4 level are $0.955,0.959$ and 0.815 , respectively. HE4, human epididymis protein 4 ; S-, serum; U-, urine; R-HE4, ratio of urinary-to-serum HE4 level.

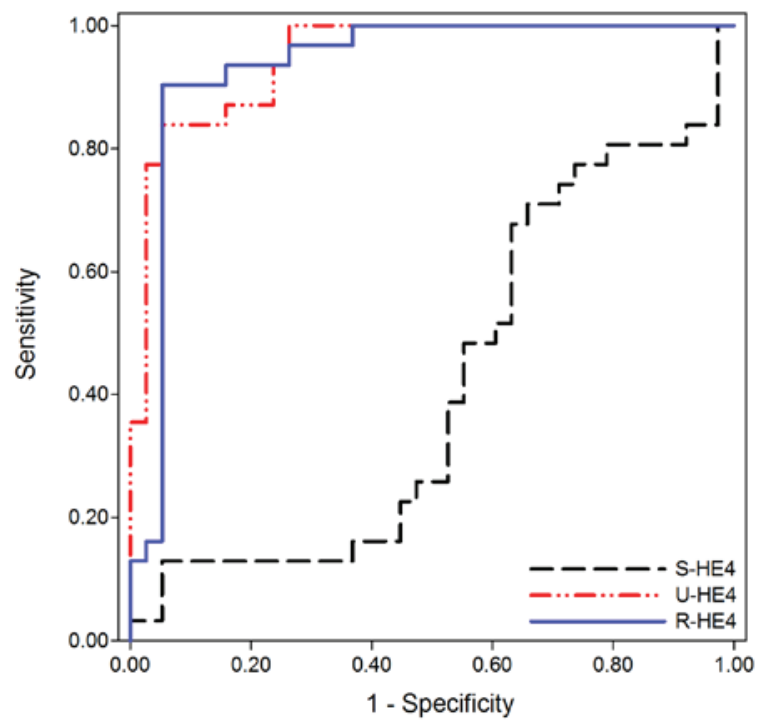

Figure 3. Receiver operating characteristic curves of HE4 for differentiating patients with ovarian cancer from chronic kidney disease patients. The area under the curve of S-HE4, U-HE4 and R-HE4 are 0.416, 0.948 and 0.915 , respectively. HE4, human epididymis protein 4; S-, serum; U-, urine; R-HE4, ratio of urinary-to-serum HE4 level.

levels detected in the $\mathrm{HC}$ group, and the study indicated that the S-HE4 level in the CKD group was higher than that in the OC group. The present study and that of Lv et al (17) indicate that the diagnosis of $\mathrm{OC}$ on the basis of S-HE4 levels may be problematic in patients who suffer from CKD.

Hellstrom et al (19) described high levels of U-HE4 in patients with OC. Macuks et al (18) reported that OC patients had higher urinary concentrations of HE4 than patients with benign ovarian tumors, and U-HE4 had comparable accuracy with S-HE4 in differentiating malignant ovarian tumors from benign disease (18). Similarly, the present study demonstrated that the U-HE4 level in the OC group was significantly higher

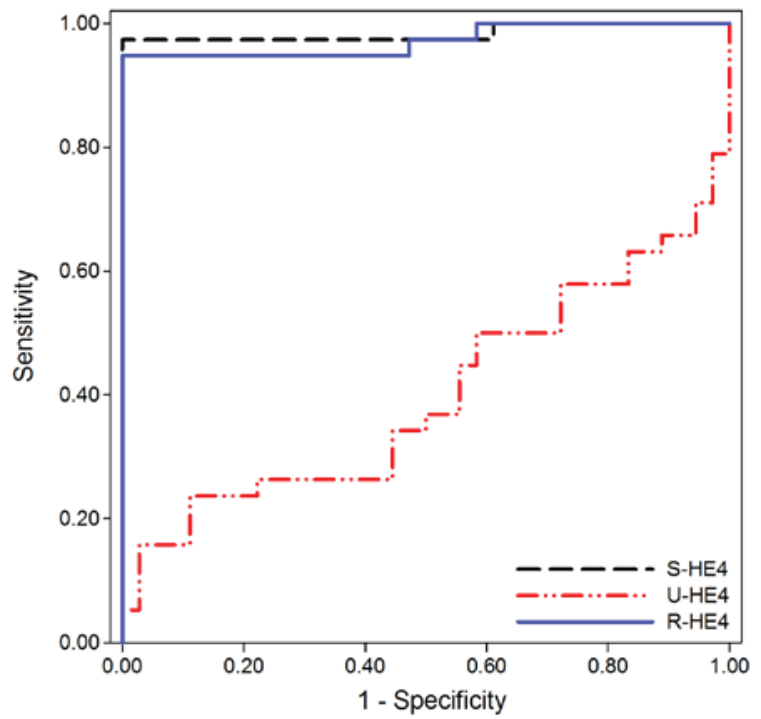

Figure 4. Receiver operating characteristics curves of HE4 for differentiating chronic kidney disease patients from healthy control subjects. The area under the curve of S-HE4, U-HE4 and R-HE4 are 0.984, 0.399 and 0.972, respectively. HE4, human epididymis protein 4; S-, serum; U-, urine; R-HE4, ratio of urinary-to-serum HE4 level.

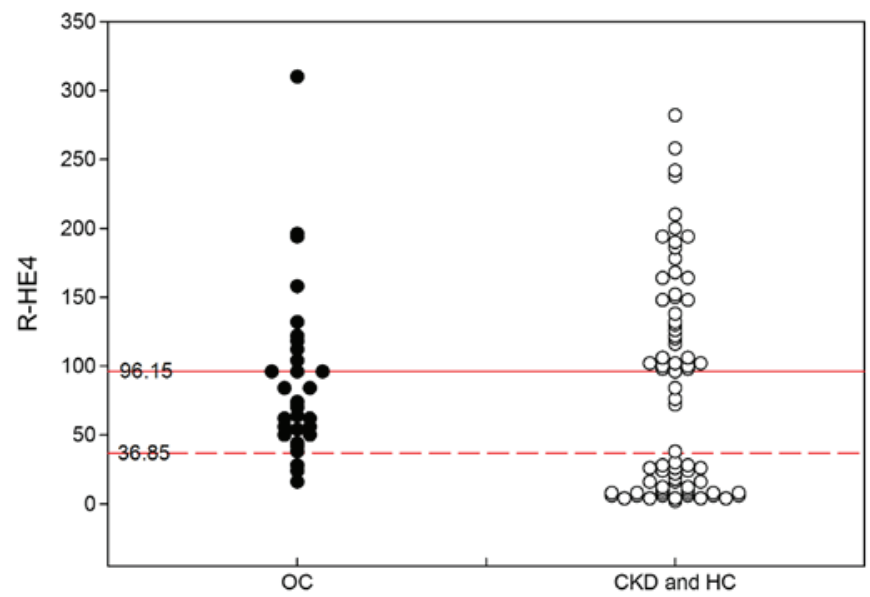

Figure 5. Diagnostic performance of R-HE4 in differentiating OC patient from non-OC objects (HC and CKD). R-HE4 provides a sensitivity of $82.6 \%$ and a specificity of $85.4 \%$ in OC diagnosis based on a diagnosis interval from 36.85 to 96.15 . R-HE4, ratio of urinary-to-serum HE4 level; OC, ovarian cancer; CKD, chronic kidney disease; HC, healthy control. The non-OC objects refer to $\mathrm{HC}$ and $\mathrm{CKD}$ subjects.

than that in the CKD and $\mathrm{HC}$ groups $(\mathrm{P}<0.001)$, and there was no significant difference in U-HE4 levels between the CKD patients and the $\mathrm{HC}$ group. These results indicate that U-HE4 level presented superior diagnostic efficacy in differentiating OC from CKD when compared with that of S-HE4 level. In the analysis of the R-HE4 diagnostic performance, there were significant differences regarding the R-HE4 among the OC and $\mathrm{CKD}$ groups, and the $\mathrm{HC}$ group $(\mathrm{HC}>\mathrm{OC}>\mathrm{CKD} ; \mathrm{P}<0.01)$. This result implies that R-HE4 maybe a candidate diagnostic marker in differentiating OC from CKD and HC.

Macuks et al compared the diagnostic performances of Sand U-HE4 levels (18). The study concluded that urine sample was an acceptable alternative for HE4 measurement, but S-HE4 measurement (AUC $=0.868$ ) was more accurate than 
U-HE4 measurement (AUC $=0.856)$ for the discrimination of patients with benign and malignant diseases. In the study by Hellstrom etal (19), the ratio of urinary HE4 to urinary creatinine presented a very high diagnostic accuracy for diagnosis of $\mathrm{OC}$ (AUC=0.969). The present study demonstrated that the AUCs of R-HE4 reached 0.935 and 0.815 in differentiating OC from CKD and HC, respectively. Furthermore, the AUC of R-HE4 was as high as 0.972 when distinguishing CKD from HC. While ROC analysis demonstrated that S-HE4 could not differentiate OC from CKD, U-HE4 could not distinguish CKD from HC. All of the data from this study indicated that R-HE4 demonstrated good diagnostic performance in differentiating OC from CKD and in differentiating CKD from HC. Thus, the clinical diagnosis of OC should be considered if the R-HE4 is between 36.85 and 96.15 (providing a sensitivity of $82.6 \%$ and a specificity of $85.4 \%$ ). Furthermore, the clinical diagnosis of CKD should be considered if R-HE4 is $<36.85$. Otherwise, the individual should be considered healthy.

There were various limitations of the present study. As a result of the difficulty in recruiting patients who simultaneously suffer from OC and CKD, the diagnostic efficacy of R-HE4 for these patients was not investigated. Numerous studies have confirmed that patients with OC have high levels of S-HE4 and patients with CKD have low levels of U-HE4. Therefore, it is reasonably speculated that the lowest R-HE4 exists in patients who simultaneously suffer from OC and CKD, as compared with $\mathrm{OC}$ and CKD patients, and $\mathrm{HC}$ subjects. In addition, the sample size of the current study was small, consisting of only 31 OC patients and 38 CKD patients.

In conclusion, S- and U-HE4 levels and R-HE4 were analyzed in $\mathrm{OC}$ and CKD patients, and $\mathrm{HC}$ subjects. Results demonstrated that OC patients had higher R-HE4 than patients with CKD and lower R-HE4 than the HC subjects. Thus, R-HE4 serves as an effective diagnostic marker for differentiating OC from CKD and HC. When R-HE4 is between 36.85 and 96.15, a clinical diagnosis of OC should be considered. The combined determination of S-and U-HE4 levels facilitates the diagnosis of OC.

\section{Acknowledgements}

The authors would like to thank the Department of Gynecology for providing the data from medical records. The authors would also like to thank the study subjects for their participation in the present study.

\section{References}

1. Jayson GC, Kohn EC, Kitchener HC and Ledermann JA: Ovarian cancer. Lancet 384: 1376-1388, 2014.

2. Bristow RE, Chang J,Ziogas A, Randall LM and Anton-Culver H: High-volume ovarian cancer care: Survival impact and disparities in access for advanced-stage disease. Gynecol Oncol 132: 403-410, 2014

3. Hamed EO, Ahmed H, Sedeek OB, Mohammed AM, Abd-Alla AA and Abdel Ghaffar HM: Significance of HE4 estimation in comparison with CA125 in diagnosis of ovarian cancer and assessment of treatment response. Diagn Pathol 8: 11, 2013.

4. Kappelmayer J, Antal-Szalmás P and Nagy B Jr: Human epididymis protein 4 (HE4) in laboratory medicine and an algorithm in renal disorders. Clin Chim Acta 438: 35-42, 2015.

5. Speeckaert MM, Speeckaert R and Delanghe JR: Human epididymis protein 4 in cancer diagnostics: A promising and reliable tumor marker. Adv Clin Chem 59: 1-21, 2013.
6. Terlikowska KM, Dobrzycka B, Witkowska AM, Mackowiak-Matejczyk B, Sledziewski TK, Kinalski M and Terlikowski SJ: Preoperative HE4, CA125 and ROMA in the differential diagnosis of benign and malignant adnexal masses. J Ovarian Res 9: 43, 2016.

7. Wei SU, Li H and Zhang B: The diagnostic value of serum HE4 and CA-125 and ROMA index in ovarian cancer. Biomed Rep 5: 41-44, 2016.

8. Xu Y, Zhong R, He J, Ding R, Lin H, Deng Y, Zhou L, Li X, Jiang J, Bao Y, et al: Modification of cut-off values for HE4, CA125 and the ROMA algorithm for early-stage epithelial ovarian cancer detection: Results from 1021 cases in South China. Clin Biochem 49: 32-40, 2016.

9. Zhang P, Wang C, Cheng L, Zhang P, Guo L, Liu W, Zhang Z, Huang Y, Ou Q, Wen X, et al: Development of a multi-marker model combining HE4, CA125, progesterone, and estradiol for distinguishing benign from malignant pelvic masses in postmenopausal women. Tumour Biol 37: 2183-2191, 2016.

10. Liao JB, Yip YY, Swisher EM, Agnew K, Hellstrom KE and Hellstrom I: Detection of the HE4 protein in urine as a biomarker for ovarian neoplasms: Clinical correlates. Gynecol Oncol 137: 430-435, 2015.

11. Wan J, Wang Y, Cai G, Liang J, Yue C, Wang F, Song J, Wang J, Liu M, Luo J, et al: Elevated serum concentrations of HE4 as a novel biomarker of disease severity and renal fibrosis in kidney disease. Oncotarget 7: 67748-67759, 2016.

12. Mckinnon B, Mueller MD, Nirgianakis K and Bersinger NA: Comparison of ovarian cancer markers in endometriosis favours HE4 over CA125. Mol Med Rep 12: 5179-5184, 2015.

13. Anton C, Carvalho FM, Oliveira EI, Maciel GA, Baracat EC and Carvalho JP: A comparison of CA125, HE4, risk ovarian malignancy algorithm (ROMA), and risk malignancy index (RMI) for the classification of ovarian masses. Clinics (Sao Paulo) 67: 437-441, 2012.

14. Nagy B Jr, Krasznai ZT, Balla H, Csobán M, Antal-Szalmás P, Hernádi $Z$ and Kappelmayer J: Elevated human epididymis protein 4 concentrations in chronic kidney disease. Ann Clin Biochem 49: 377-380, 2012.

15. Piek A, Meijers WC, Schroten NF, Gansevoort RT, de Boer RA and Sillje HH: HE4 Serum levels are associated with heart failure severity in patients with chronic heart failure. J Card Fail 23: 12-19, 2017.

16. Yang Z, Zhang Z, Qin B, Wu P, Zhong R, Zhou L and Liang Y: Human Epididymis Protein 4: A novel biomarker for Lupus nephritis and chronic kidney disease in systemic Lupus erythematosus. J Clin Lab Anal 30: 897-904, 2016.

17. Lv YW, Yang L, Zhang M, Jiang LH, Niu JH, Hou J and Cui XH: Increased human epididymis protein 4 in benign gynecological diseases complicated with chronic renal insufficiency patients. Genet Mol Res 14: 2156-2161, 2015.

18. Macuks R, Baidekalna I and Donina S: Urinary concentrations of human epidydimis secretory protein $4(\mathrm{He} 4)$ in the diagnosis of ovarian cancer: A case - control study. Asian Pac J Cancer Prev 13: 4695-4698, 2012.

19. Hellstrom I, Heagerty PJ, Swisher EM, Liu P, Jaffar J, Agnew K and Hellstrom KE: Detection of the HE4 protein in urine as a biomarker for ovarian neoplasms. Cancer Lett 296: 43-48, 2010.

20. FIGO Committee on Gynecologic Oncology: FIGO staging for carcinoma of the vulva, cervix, and corpus uteri. Int J Gynaecol Obstet 125: 97-98, 2014.

21. Kidney Disease Improving Global Outcomes (KDIGO) CKD Work Group: KDIGO 2012 clinical practice guideline for the evaluation and management of chronic kidney disease. Kidney inter 3: 1-150. 2013.

22. Levey AS, Stevens LA, Schmid CH, Zhang YL, Castro AF III, Feldman HI, Kusek JW, Eggers P, Van Lente F, Greene T, and Coresh J; CKD-EPI (Chronic Kidney Disease Epidemiology Collaboration): A new equation to estimate glomerular filtration rate. Ann Intern Med 150: 604-612, 2009.

23. Ferraro S, Braga F, Lanzoni M, Boracchi P, Biganzoli EM and Panteghini M: Serum human epididymis protein 4 vs carbohydrate antigen 125 for ovarian cancer diagnosis: A systematic review. J Clin Pathol 66: 273-281, 2013.

24. Gündüz UR, Gunaldi M, Isiksacan N, Gündüz S, Okuturlar Y and Kocoglu H: A new marker for breast cancer diagnosis, human epididymis protein 4: A preliminary study. Mol Clin Oncol 5: 355-360, 2016.

25. Lamy PJ, Plassot C and Pujol JL: Serum HE4: An independent prognostic factor in non-small cell lung cancer. PLoS One 10: e0128836, 2015. 